\title{
On new integral inequalities for $m$-logarithmically-convex functions
}

\author{
Huriye Kadakal \\ Institute of Science, Ordu University, Ordu, Turkey \\ Received: 4 December 2017, Accepted: 25 December 2017 \\ Published online: 25 September 2018.
}

\begin{abstract}
In this work, by using an integral identity we obtain several new inequalities for $m$-time differentiable $m$-logarithmicallyconvex functions. We should especially mention that the results obtained in special cases coincide with the well-known results in the literature.
\end{abstract}

Keywords: Convex function, logarithmically-Convex function, $m$-Logarithmically-Convex function.

\section{Introduction}

In this study, we establish some new inequalities for functions whose $n$th derivatives in absolute value are $m$-logarithmically-convex. The theory of convex analysis has emerged as one of the most interesting and useful field of mathematics and the other sciences in last few decades. For some inequalities, generalizations and applications concerning convexity see [7,9-11,13,17,18]. Recently, in the literature there are so many papers about $n$-times differentiable functions on several kinds of convexities. In references [3,6,8,14-16,19,25], readers can find some results about this issue. Logarithmically convex (log-convex) functions are of interest in many areas of mathematics and the other science. They have been found to play an important role in the theory of special functions and mathematical statistics (see, e.g., [5], [23]). Many papers have been written by a number of mathematicians concerning inequalities for different classes of logarithmically-convex functions see for instance the recent papers [1,2,8,20-22,24] and the references within these papers.

Definition 1. A function $f: I \subseteq \mathbb{R} \rightarrow \mathbb{R}$ is said to be convex if the inequality

$$
f(t x+(1-t) y) \leq t f(x)+(1-t) f(y)
$$

is valid for all $x, y \in I$ and $t \in[0,1]$. If this inequality reverses, then $f$ is said to be concave on interval I $\neq \emptyset$. This definition is well known in the literature.

Definition 2. A positive function $f$ is called logarithmically-convex on a real interval $I=[a, b]$, if for all $x, y \in[a, b]$ and $t \in[0,1]$,

$$
f(t x+(1-t) y) \leq[f(x)]^{t}[f(y)]^{1-t} .
$$

If $f$ is a positive logarithmically-concave function, then the inequality is reversed.

Equivalently, a function $f$ is logarithmically-convex on $I$ if $f$ is positive and $\log f$ is convex on $I$. Also, if $f>0$ and $f^{\prime \prime}$ exists on I, then $f$ is logarithmically-convex if and only if $f f^{\prime \prime}-\left(f^{\prime}\right)^{2} \geq 0$. Let $0<a<b$. We will use the following notations throughout this paper. 
(i) Arithmetic Mean:

$$
A(a, b)=\frac{a+b}{2}, \forall a, b \in \mathbb{R}^{+}
$$

(ii) Logarithmic Mean:

$$
L(a, b)=\frac{b-a}{\ln b-\ln a}, \quad \forall a, b \in \mathbb{R}^{+}, a \neq b
$$

(iii) Generalized Logarithmic Mean:

$$
L_{p}(a, b)=\left(\frac{b^{p+1}-a^{p+1}}{(p+1)(b-a)}\right)^{\frac{1}{p}}, \forall a \neq b, p \in \mathbb{R}, p \neq-1,0
$$

(iv) Exponential Mean:

$$
E(a, b)=\frac{e^{a}-e^{b}}{a-b}, a \neq b \text { and } E(a, a)=a
$$

Throughout this paper, we will use the following natation for shortness:

$$
\mu_{f}=\mu_{f}(a, b, m, n, q)=\frac{\left|f^{(n)}(a)\right|^{q}}{\left|f^{(n)}\left(\frac{b}{m}\right)\right|^{q m}}
$$

where, $f: I \subseteq[0, \infty) \rightarrow(0, \infty)$ be $n$-times differentiable function on $I^{\circ}$ and $a, \frac{b}{m} \in I^{\circ}$ with $0 \leq a<b<\infty, m \in(0,1]$ and $q>1$.

Definition 3. [4] A positive $f:[0, b] \rightarrow(0, \infty)$ is said to be m-logarithmically-convex if the inequality

$$
f(t x+m(1-t) y) \leq[f(x)]^{t}[f(y)]^{m(1-t)} .
$$

holds for all $x, y \in[a, b], m \in(0,1]$ and $t \in[0,1]$.

Obviously, if putting $m=1$ in the above definition, then $f$ is just the ordinary logarithmically convex function on $[a, b]$.

\section{Main results}

We will use the following Lemma [19] to obtain our main results.

Lemma 1. Let $f: I \subseteq \mathbb{R} \rightarrow \mathbb{R}$ be n-times differentiable mapping on $I^{\circ}$ for $n \in \mathbb{N}$ and $f^{(n)} \in L[a, b]$, where a,b $\in I^{\circ}$ with $a<b$, we have the identity

$$
\int_{k=0}^{n-1}(-1)^{k}\left(\frac{f^{(k)}(b) b^{k+1}-f^{(k)}(a) a^{k+1}}{(k+1) !}\right)-\int_{a}^{b} f(x) d x=\frac{(-1)^{n+1}}{n !} \int_{a}^{b} x^{n} f^{(n)}(x) d x .
$$

where an empty sum is understood to be nil.

Theorem 1. For $\forall n \in \mathbb{N}$; let $f: I \subseteq[0, \infty) \rightarrow(0, \infty)$ be $n$-times differentiable function on $I^{\circ}$ and $a, \frac{b}{m} \in I^{\circ}$ with $0 \leq a<b<\infty$ and $m \in(0,1]$. If $f^{(n)} \in L[a, b]$ and $\left|f^{(n)}\right|^{q}$ for $q>1$ is m-logarithmically-convex on $[a, b]$, then the following inequality holds:

$$
\left|\int_{k=0}^{n-1}(-1)^{k}\left(\frac{f^{(k)}(b) b^{k+1}-f^{(k)}(a) a^{k+1}}{(k+1) !}\right)-\int_{a}^{b} f(x) d x\right| \leq \frac{1}{n !}(b-a) L_{n p}^{n}(a, b) L^{\frac{1}{q}}\left(\left|f^{(n)}\left(\frac{b}{m}\right)\right|^{q m},\left|f^{(n)}(a)\right|^{q}\right)
$$

where $\frac{1}{p}+\frac{1}{q}=1, L$ and $L_{p}$ are logarithmic and generalized logarithmic means, respectively. 
Proof. Since $\left|f^{(n)}\right|^{q}$ for $q>1$ is $m$-logarithmically-convex on $[a, b]$, using Lemma 1, the Hölder integral inequality and

$$
\left|f^{(n)}(x)\right|^{q}=\left|f^{(n)}\left(\frac{b-x}{b-a} a+m \frac{x-a}{b-a} \frac{b}{m}\right)\right|^{q} \leq\left[\left|f^{(n)}(a)\right|^{q}\right]^{\frac{b-x}{b-a}}\left[\left|f^{(n)}\left(\frac{b}{m}\right)\right|^{q}\right]^{m\left(1-\frac{b-x}{b-a}\right)}
$$

we have

$$
\begin{aligned}
& \left|\int_{k=0}^{n-1}(-1)^{k}\left(\frac{f^{(k)}(b) b^{k+1}-f^{(k)}(a) a^{k+1}}{(k+1) !}\right)-\int_{a}^{b} f(x) d x\right| \leq \frac{1}{n !} \int_{a}^{b} x^{n}\left|f^{(n)}(x)\right| d x \\
& \leq \frac{1}{n !}\left(\int_{a}^{b} x^{n p} d x\right)^{\frac{1}{p}}\left(\int_{a}^{b}\left|f^{(n)}(x)\right|^{q} d x\right)^{\frac{1}{q}} \\
& \leq \frac{1}{n !}\left(\int_{a}^{b} x^{n p} d x\right)\left(\int_{a}^{b}\left[\left|f^{(n)}(a)\right|^{q}\right]^{\frac{1-x}{b-a}}\left[\left|f^{(n)}\left(\frac{b}{m}\right)\right|^{q}\right]^{m\left(1-\frac{b-x}{b-a}\right)} d x\right)^{\frac{1}{q}} \\
& =\frac{1}{n !}\left|f^{(n)}\left(\frac{b}{m}\right)\right|^{m}\left(\int_{a}^{b} x^{n p} d x\right)^{\frac{1}{p}}\left(\int_{a}^{b} \mu_{\left.f^{\frac{b-x}{b-a}} d x\right)^{\frac{1}{q}}}\right. \\
& =\frac{1}{n !}\left|f^{(n)}\left(\frac{b}{m}\right)\right|^{m}\left(\frac{b^{n p+1}}{n p+1}-\frac{a^{n p+1}}{n p+1}\right)^{\frac{1}{p}} \frac{b-a}{\ln \left|f^{(n)}\left(\frac{b}{m}\right)\right|^{q m}-\ln \left|f^{(n)}(a)\right|^{q}}\left(1-\mu_{f}\right)^{\frac{1}{q}} \\
& =\frac{1}{n !}(b-a)\left[\frac{b^{n p+1}-a^{n p+1}}{(n p+1)(b-a)}\right]^{\frac{1}{p}} \frac{\left|f^{(n)}\left(\frac{b}{m}\right)\right|^{q m}-\left|f^{(n)}(a)\right|^{q}}{\ln \left|f^{(n)}\left(\frac{b}{m}\right)\right|^{q m}-\ln \left|f^{(n)}(a)\right|^{q}} \\
& =\frac{1}{n !}(b-a) L_{n p}^{n}(a, b) L^{\frac{1}{q}}\left(\left|f^{(n)}\left(\frac{b}{m}\right)\right|^{q m},\left|f^{(n)}(a)\right|^{q}\right)
\end{aligned}
$$

This completes the proof of theorem.

Remark. If we take $m=1$ in Theorem 1, then the results coincide with [12].

Corollary 1. Under the conditions Theorem 1 for $n=1$ we have the following inequality.

$$
\left|\frac{f(b) b-f(a) a}{b-a}-\frac{1}{b-a} \int_{a}^{b} f(x) d x\right| \leq L_{p}(a, b) L^{\frac{1}{q}}\left(\left|f^{\prime}\left(\frac{b}{m}\right)\right|^{q m},\left|f^{\prime}(a)\right|^{q}\right) .
$$

Remark. If we take $m=1$ in Corollary 1, then the results coincide with [12].

Proposition 1. Let $a, b \in(0, \infty)$ with $a<b, p, q>1$ with $\frac{1}{p}+\frac{1}{q}=1$ and we have

$$
\left|q e^{\frac{b}{q}}-(q-a) E\left(\frac{a}{q}, \frac{b}{q}\right)\right| \leq L_{p}(a, b) E^{\frac{1}{q}}(a, b)
$$

Proof.Under the assumption of the Proposition, let $f(x)=q e^{\frac{x}{q}}, x \in(0, \infty)$. Then

$$
\left|f^{\prime}(x)\right|=e^{\frac{x}{q}}
$$

is $m$-log-convex on $(0, \infty)$ and the result follows directly from Corollary 1.

Theorem 2. For $\forall n \in \mathbb{N}$; let $f: I \subseteq[0, \infty) \rightarrow(0, \infty)$ be n-times differentiable function on $I^{\circ}$ and $a, \frac{b}{m} \in I^{\circ}$ with $0 \leq a<b<\infty$ and $m \in(0,1]$. If $f^{(n)} \in L[a, b]$ and $\left|f^{(n)}\right|^{q}$ for $q \geq 1$ is m-logarithmically-convex on $[a, b]$, then the following inequality 
holds.

$$
\left|\int_{k=0}^{n-1}(-1)^{k}\left(\frac{f^{(k)}(b) b^{k+1}-f^{(k)}(a) a^{k+1}}{(k+1) !}\right)-\int_{a}^{b} f(x) d x\right| \leq \frac{1}{n !}(b-a)\left|f^{(n)}\left(\frac{b}{m}\right)\right|^{m} L_{n}^{n\left(\frac{q-1}{q}\right)}(a, b) M^{\frac{1}{q}}
$$

where $M=\int_{0}^{1}[b-(b-a) t]^{n} \mu_{f}^{t} d t$ and $L_{n}$ is generalized logarithmic mean.

Proof. From Lemma 1 and Power-mean integral inequality, we obtain

$$
\begin{aligned}
& \left|\int_{k=0}^{n-1}(-1)^{k}\left(\frac{f^{(k)}(b) b^{k+1}-f^{(k)}(a) a^{k+1}}{(k+1) !}\right)-\int_{a}^{b} f(x) d x\right| \leq \frac{1}{n !} \int_{a}^{b} x^{n}\left|f^{(n)}(x)\right| d x \\
& \leq \frac{1}{n !}\left(\int_{a}^{b} x^{n} d x\right)^{1-\frac{1}{q}}\left(\int_{a}^{b} x^{n}\left|f^{(n)}(x)\right|^{q} d x\right)^{\frac{1}{q}} \\
& \leq \frac{1}{n !}\left(\int_{a}^{b} x^{n} d x\right)^{1-\frac{1}{q}}\left(\int_{a}^{b} x^{n}\left[\left|f^{(n)}(a)\right|^{q}\right]^{\frac{b-x}{b-a}}\left[\left|f^{(n)}\left(\frac{b}{m}\right)\right|^{q}\right]^{m\left(1-\frac{b-x}{b-a}\right)} d x\right)^{\frac{1}{q}} \\
& =\frac{1}{n !}\left|f^{(n)}\left(\frac{b}{m}\right)\right|^{m}\left(\int_{a}^{b} x^{n} d x\right)^{1-\frac{1}{q}}\left(\int_{a}^{b} x^{n} \mu_{f^{\frac{b-x}{b-a}}} d x\right)^{\frac{1}{q}} \\
& =\frac{1}{n !}(b-a)^{\frac{1}{q}}\left|f^{(n)}\left(\frac{b}{m}\right)\right|^{m}\left(\int_{a}^{b} x^{n} d x\right)^{1-\frac{1}{q}}\left(\int_{0}^{1}[b-(b-a) t]^{n} \mu_{f}^{t} d t\right)^{\frac{1}{q}} \\
& =\frac{1}{n !}(b-a)^{\frac{1}{q}}\left|f^{(n)}\left(\frac{b}{m}\right)\right|^{m}\left(\frac{b^{n+1}-a^{n+1}}{n+1}\right)^{1-\frac{1}{q}} M^{\frac{1}{q}} \\
& =\frac{1}{n !}\left|f^{(n)}\left(\frac{b}{m}\right)\right|^{m}(b-a)^{\frac{1}{q}}(b-a)^{1-\frac{1}{q}}\left[\frac{b^{n+1}-a^{n+1}}{(n+1)(b-a)}\right]^{1-\frac{1}{q}} M^{\frac{1}{q}} \\
& =\frac{1}{n !}(b-a)\left|f^{(n)}\left(\frac{b}{m}\right)\right|^{m} L_{n}^{n\left(\frac{q-1}{q}\right)}(a, b) M^{\frac{1}{q}} \text {. }
\end{aligned}
$$

Remark. If we take $m=1$ in Theorem 2, then the results coincide with [12].

Corollary 2. Under the conditions Theorem 2 for $n=1$ we have the following inequality.

$$
\left|\frac{f(b) b-f(a) a}{b-a}-\frac{1}{b-a} \int_{a}^{b} f(x) d x\right| \leq A^{1-\frac{1}{q}}(a, b) \frac{b\left|f^{\prime}\left(\frac{b}{m}\right)\right|^{q m}-a\left|f^{\prime}(a)\right|^{q}}{\ln \left|f^{\prime}\left(\frac{b}{m}\right)\right|^{q m}-\ln \left|f^{\prime}(a)\right|^{q}}-(b-a) \frac{L\left(\left|f^{\prime}\left(\frac{b}{m}\right)\right|^{q m},\left|f^{\prime}(a)\right|^{q}\right)^{\frac{1}{q}}}{\ln \left|f^{\prime}\left(\frac{b}{m}\right)\right|^{q m}-\ln \left|f^{\prime}(a)\right|^{q}}
$$

Remark. If we take $m=1$ in Corollary 2, then the results coincide with [12].

Proposition 2. Let $a, b \in(0, \infty)$ with $a<b, q>1$ and, we have

$$
\left|q^{\frac{b}{q}}+(a-q) E\left(\frac{b}{q}, \frac{a}{q}\right)\right| \leq A^{1-\frac{1}{q}}(a, b)\left[e^{b}+(a-1) E(a, b)\right]^{\frac{1}{q}} .
$$

Proof. The result follows directly from Corollary 2 for the function $f(x)=q e^{\frac{x}{q}}, x \in(0, \infty)$.

Corollary 3. Under the conditions Theorem 2 for $q=1$ we have the following inequality.

$$
\left|\int_{k=0}^{n-1}(-1)^{k}\left(\frac{f^{(k)}(b) b^{k+1}-f^{(k)}(a) a^{k+1}}{(k+1) !}\right)-\int_{a}^{b} f(x) d x\right| \leq \frac{1}{n !}(b-a)\left|f^{(n)}\left(\frac{b}{m}\right)\right|^{m} M
$$


Remark. If we take $m=1$ in Corollary 3 , then the results coincide with [12].

Corollary 4. Under the conditions Theorem 2 for $m=1$ we have the following inequality.

$$
\left|\int_{k=0}^{n-1}(-1)^{k}\left(\frac{f^{(k)}(b) b^{k+1}-f^{(k)}(a) a^{k+1}}{(k+1) !}\right)-\int_{a}^{b} f(x) d x\right| \leq \frac{1}{n !}(b-a)\left|f^{(n)}(b)\right| M .
$$

Theorem 3. For $\forall n \in \mathbb{N}$; let $f: I \subseteq[0, \infty) \rightarrow(0, \infty)$ be n-times differentiable function on $I^{\circ}$ and $a, \frac{b}{m} \in I^{\circ}$ with $0 \leq a<b<\infty$ and $m \in(0,1]$. If $f^{(n)} \in L[a, b]$ and $\left|f^{(n)}\right|^{q}$ for $q>1$ is $m$-logarithmically-convex on $[a, b]$. then the following inequality holds.

$$
\begin{aligned}
& \left|\int_{k=0}^{n-1}(-1)^{k}\left(\frac{f^{(k)}(b) b^{k+1}-f^{(k)}(a) a^{k+1}}{(k+1) !}\right)-\int_{a}^{b} f(x) d x\right| \\
& \leq \frac{1}{n !}(b-a) \sum_{p\left(n-\frac{1}{q}\right)}^{\frac{q n-1}{q}}(a, b) \frac{b\left|f^{\prime}\left(\frac{b}{m}\right)\right|^{q m}-a\left|f^{\prime}(a)\right|^{q}}{\ln \left|f^{\prime}\left(\frac{b}{m}\right)\right|^{q m}-\ln \left|f^{\prime}(a)\right|^{q}}-(b-a) \frac{L\left(\left|f^{\prime}\left(\frac{b}{m}\right)\right|^{q m},\left|f^{\prime}(a)\right|^{q}\right)^{\frac{1}{q}}}{\ln \left|f^{\prime}\left(\frac{b}{m}\right)\right|^{q m}-\ln \left|f^{\prime}(a)\right|^{q}}
\end{aligned}
$$

where $\frac{1}{p}+\frac{1}{q}=1, M=\int_{0}^{1}[b-(b-a) t]^{n} \mu_{f}^{t} d t$ and $L_{p}$ is generalized logarithmic mean.

Proof. Since $\left|f^{(n)}\right|^{q}$ for $q>1$ is $m$-logarithmically-convex on $[a, b]$, using Lemma 1 and the Hölder integral inequality, we obtain the following inequality.

$$
\begin{aligned}
& \left|\int_{k=0}^{n-1}(-1)^{k}\left(\frac{f^{(k)}(b) b^{k+1}-f^{(k)}(a) a^{k+1}}{(k+1) !}\right)-\int_{a}^{b} f(x) d x\right| \leq \frac{1}{n !} \int_{a}^{b} x^{n-\frac{1}{q}} \cdot x^{\frac{1}{q}}\left|f^{(n)}(x)\right| d x \\
& \leq \frac{1}{n !}\left(\int_{a}^{b}\left(x^{n-\frac{1}{q}}\right)^{p} d x\right)^{\frac{1}{p}}\left(\int_{a}^{b}\left(x^{\frac{1}{q}}\right)^{q}\left|f^{(n)}(x)\right|^{q} d x\right)^{\frac{1}{q}} \\
& \leq \frac{1}{n !}\left(\int_{a}^{b} x^{p \frac{q n-1}{q}} d x\right)^{\frac{1}{p}}\left(\int_{a}^{b} x\left[\left|f^{(n)}(a)\right|^{q}\right]^{\frac{b-x}{b-a}}\left[\left|f^{(n)}\left(\frac{b}{m}\right)\right|^{q}\right]^{m\left(1-\frac{b-x}{b-a}\right)} d x\right)^{\frac{1}{q}} \\
& =\frac{1}{n !}\left|f^{(n)}\left(\frac{b}{m}\right)\right|^{m}\left(\int_{a}^{b} x^{p \frac{q n-1}{q}} d x\right)^{\frac{1}{p}}\left(\int_{a}^{b} x \mu_{f^{\frac{b-x}{b-a}}} d x\right)^{\frac{1}{q}} \\
& =\frac{1}{n !}\left|f^{(n)}\left(\frac{b}{m}\right)\right|^{m}(b-a)^{\frac{1}{p}}\left[\frac{b^{p \frac{q n-1}{q}+1}-a^{p \frac{q n-1}{q}+1}}{\left(p \frac{q n-1}{q}+1\right)(b-a)}\right]^{\frac{1}{p}} \\
& \times \frac{(b-a)\left[a\left|f^{(n)}(a)\right|^{q}-b\left|f^{(n)}\left(\frac{b}{m}\right)\right|^{q m}\right]}{\ln \left|f^{(n)}(a)\right|^{q}-\ln \left|f^{(n)}\left(\frac{b}{m}\right)\right|^{q m}}-\frac{(b-a)^{2} L\left(\left|f^{(n)}(a)\right|^{q},\left|f^{(n)}\left(\frac{b}{m}\right)\right|^{q m}\right)^{\frac{1}{q}}}{\ln \left|f^{(n)}(a)\right|^{q}-\ln \left|f^{(n)}\left(\frac{b}{m}\right)\right|^{q m}} \\
& =\frac{1}{n !}(b-a)\left[\frac{b^{p \frac{q n-1}{q}+1}-a^{p \frac{q n-1}{q}+1}}{\left(p \frac{q n-1}{q}+1\right)(b-a)}\right]^{\frac{1}{p}} \\
& \times \frac{a\left|f^{(n)}(a)\right|^{q}-b\left|f^{(n)}\left(\frac{b}{m}\right)\right|^{q m}}{\ln \left|f^{(n)}(a)\right|^{q}-\ln \left|f^{(n)}\left(\frac{b}{m}\right)\right|^{q m}}-\frac{(b-a) L\left(\left|f^{(n)}(a)\right|^{q},\left|f^{(n)}\left(\frac{b}{m}\right)\right|^{q m}\right)^{\frac{1}{q}}}{\ln \left|f^{(n)}(a)\right|^{q}-\ln \left|f^{(n)}\left(\frac{b}{m}\right)\right|^{q m}} \\
& =\frac{1}{n !}(b-a) L_{p\left(n-\frac{1}{q}\right)}^{\frac{q n-1}{q}}(a, b) \frac{b\left|f^{\prime}\left(\frac{b}{m}\right)\right|^{q m}-a\left|f^{\prime}(a)\right|^{q}}{\ln \left|f^{\prime}\left(\frac{b}{m}\right)\right|^{q m}-\ln \left|f^{\prime}(a)\right|^{q}}-\frac{(b-a) L\left(\left|f^{\prime}\left(\frac{b}{m}\right)\right|^{q m},\left|f^{\prime}(a)\right|^{q}\right)^{\frac{1}{q}}}{\ln \left|f^{\prime}\left(\frac{b}{m}\right)\right|^{q m}-\ln \left|f^{\prime}(a)\right|^{q}} .
\end{aligned}
$$


Remark. If we take $m=1$ in Theorem 3, then the results coincide with [12].

Corollary 5. Under the conditions Theorem 3 for $n=1$ we have the following inequality.

$$
\left|\frac{f(b) b-f(a) a}{b-a}-\frac{1}{b-a} \int_{a}^{b} f(x) d x\right| \leq A^{\frac{1}{p}}(a, b) \frac{b\left|f^{\prime}\left(\frac{b}{m}\right)\right|^{q m}-a\left|f^{\prime}(a)\right|^{q}}{\ln \left|f^{\prime}\left(\frac{b}{m}\right)\right|^{q m}-\ln \left|f^{\prime}(a)\right|^{q}}-\frac{(b-a) L\left(\left|f^{\prime}\left(\frac{b}{m}\right)\right|^{q m},\left|f^{\prime}(a)\right|^{q}\right)^{\frac{1}{q}}}{\ln \left|f^{\prime}\left(\frac{b}{m}\right)\right|^{q m}-\ln \left|f^{\prime}(a)\right|^{q}} .
$$

where A is arithmetic mean.

Remark. If we take $m=1$ in Corollary 5, then the results coincide with [12].

Corollary 6. Under the conditions Theorem 3 for $n=1$ and $m=1$ we have the following inequality

$$
\left|\frac{f(b) b-f(a) a}{b-a}-\frac{1}{b-a} \int_{a}^{b} f(x) d x\right| \leq A^{\frac{1}{p}}(a, b) \frac{b\left|f^{\prime}(b)\right|^{q}-a\left|f^{\prime}(a)\right|^{q}}{\ln \left|f^{\prime}(b)\right|^{q}-\ln \left|f^{\prime}(a)\right|^{q}}-\frac{(b-a) L\left(\left.\left|f^{\prime}(b)\right|\right|^{q},\left|f^{\prime}(a)\right|^{q}\right)^{\frac{1}{q}}}{\ln \left|f^{\prime}(b)\right|^{q}-\ln \left|f^{\prime}(a)\right|^{q}}
$$

which coincides with the result in [12].

Proposition 3. Let $a, b \in(0, \infty)$ with $a<b, p, q>1, \frac{1}{p}+\frac{1}{q}=1$, we have

$$
\left|q e^{\frac{b}{q}}+(a-q) E\left(\frac{a}{q}, \frac{b}{q}\right)\right| \leq A^{1-\frac{1}{q}}(a, b)\left[e^{b}+(a-1) E(a, b)\right]^{\frac{1}{q}}
$$

Proof. The result follows directly from Corollary 5 for the function $f(x)=q e^{\frac{x}{q}}, x \in(0, \infty)$.

\section{Conclusions}

In this paper, by using an integral identity we obtain some new type inequalities for $n$-time differentiable $m$-logarithmically-convex functions.

\section{Competing interests}

The authors declare that they have no competing interests.

\section{Authors' contributions}

All authors have contributed to all parts of the article. All authors read and approved the final manuscript.

\section{References}

[1] M. Alomari and M. Darus, "On The Hadamard's Inequality for Log-Convex Functions on the Coordinates”, Hindawi Publishing Corporation Journal of Inequalities and Applications, Volume 2009, Article ID 283147, 13 pages.

[2] M. A. Ardıç and M. Emin Özdemir, "Inequalities for log-convex functions vıa three times differentiability", arXiv:1405.7480v1 [math.CA] 29 May 2014.

[3] S.-P. Bai, S.-H. Wang and F. Qi, "Some Hermite-Hadamard type inequalities for n-time differentiable $(\alpha, \mathrm{m})$-convex functions", Jour. of Ineq. and Appl., 2012, 2012:267. 
[4] R.-F. Bai, F. Qi, B.-Y. Xi, "Hermite-Hadamard type inequalities for them-and ( $\alpha, \mathrm{m})$-logarithmically convex functions", Filomat 27:1 (2013), 1-7.

[5] B.C. Carlson, "Special Functions of Applied Mathematics", Academic Press, New York, 1977.

[6] P. Cerone, S.S. Dragomir, J. Roumeliotis and J. Sunde, "A new generalization of the trapezoid formula for n-time differentiable mappings and applications", Demonstratio Math., 33(4), (2000), 719-736.

[7] S.S. Dragomir and C.E.M. Pearce, "Selected Topics on Hermite-Hadamard Inequalities and Applications", RGMIA Monographs, Victoria University, 2000.

[8] S.S. Dragomir, "New jensen's type $\imath$ nequal $\imath t \imath e s$ for d $\imath$ fferent $\imath$ able log-convex functions of selfadjoınt operators $\imath$ n Hilbert spaces", Sarajevo Journal of Mathematics, Vol.7(19), (2011), 67-80.

[9] İ. İşcan, "Ostrowski type inequalities for p-convex functions”, New Trends in Mathematical Sciences, 4(3), (2016), 140-150.

[10] İ. İşcan, "Ostrowski type inequalities for p-convex functions”, New Trends in Mathematical Sciences, 4(3), (2016), 140-150.

[11] İ. İşcan and S. Turhan, "Generalized Hermite-Hadamard-Fejer type inequalities for GA-convex functions via Fractional integral", Moroccan J. Pure and Appl. Anal.(MJPAA), Vol. 2(1), (2016), 34-46.

[12] İ. İşcan, H. Kadakal, M. Kadakal, "Some new integral inequalities for n-times differentiable log-convex functions", New Trends in Mathematical Sciences, NTMSCI 5, No. 2, 10-15 (2017).

[13] İ. İşcan, H. Kadakal and M. Kadakal, "Some New Integral Inequalities for n-Times Differentiable Quasi-Convex Functions", Sigma Journal of Engineering and Natural Sciences, 35(3), 363-368, 2017.

[14] W.-D. Jiang, D.W. Niu, Y. Hua and F. Qi, "Generalizations of Hermite-Hadamard inequality to n-time differentiable function which are s-convex in the second sense", Analysis (Munich), 32(2012), 209-220.

[15] M. Kadakal, H. Kadakal and İ. İşcan "Some new integral inequalities for n-times differentiable s-convex functions in the first sense", Turkish Journal of Analysis and Number Theory, 2017, 5, 2, 63-68.

[16] H. Kadakal, M. Kadakal and İ. İşcan, "Some new integral inequalities for n-times differentiable s-convex and s-concave functions in the second sense", Mathematics and Statistic, 5(2), 94-98, 2017.

[17] M. Kunt, İ. İşcan, "On new inequalities of Hermite-Hadamard-Fejer type for GA-s-convex functions via fractional integrals ", Konuralp Journal of Mathematics, vol.4, no.1, pp.130-139, 2016.

[18] M. Kunt, İ. İşcan, N. Yazici Gözütok, "Hermite-Hadamard type inequalities for product of harmonically convex functions via Riemann-Liouville fractional integrals", Journal of Mathematical Analysis, vol.7, no.4, pp.74-82, 2016.

[19] S. Maden, H. Kadakal, M. Kadakal and İ. İşcan, "Some new integral inequalities for n-times differentiable convex and concave functions", Journal of Nonlinear Sciences and Applications, 10(12), (2017), 6141-6148.

[20] M. Mansour, M. A. Obaid, "A Generalization of Some Inequalities for the log-Convex Functions", International Mathematical Forum, 5, 2010, no. 65, 3243 - 3249.

[21] C. P. Niculescu, “The Hermite-Hadamard inequality for log-convex functions”, Nonlinear Analysis 75 (2012) $662-669$.

[22] J. Park, "Some Hermite-Hadamard-like Type Inequalities for Logarithmically Convex Functions", Int. Journal of Math. Analysis, Vol. 7, 2013, no. 45, 2217-2233.

[23] J.E. Pečarı́, F. Proschan and Y.L. Tong, "Convex Functions, Partial Orderings and Statistical Applications”, Academic Press, Boston, 1992.

[24] X. Zhang, W. Jiang, "Some properties of log-convex function and applications for the exponential function”, Computers and Mathematics with Applications 63 (2012) 1111-1116.

[25] G. S. Yang, K. L. Tseng and H. T. Wang, "A note on $\imath$ ntegral inequalittes of Hadamard type for log-convex and log-concave functions", Taiwanese Journal of Mathematics, Vol. 16, No. 2, pp. 479-496, April 2012. 